\title{
Changes in the Diagnostic Efficiency of Thyroid Fine-Needle Aspiration Biopsy during the Era of Increased Thyroid Cancer Screening in Korea
}

\author{
Young Ki Lee, MD ${ }^{1}$ \\ Kyeong Hye Park, MD2,3 \\ Young Duk Song, MD, $\mathrm{PhD}^{3}$ \\ Taemi Youk ${ }^{4,5}$ \\ Joo Young Nam, MD, $\mathrm{PhD}^{3}$ \\ Sun Ok Song, MD, PhD ${ }^{3}$ \\ Dong Yeob Shin, MD, $\mathrm{PhD}^{6}$ \\ Eun Jig Lee, MD, $P h D^{6}$
}

${ }^{1}$ Center for Thyroid Cancer, National Cancer Center, Goyang, ${ }^{2}$ Department of Medicine, Yonsei University College of Medicine, Seoul, ${ }^{3}$ Division of Endocrinology and Metabolism, Department of Internal Medicine, National Health Insurance Service Ilsan Hospital, Goyang, ${ }^{4}$ Research Institute, National Health Insurance Service Ilsan Hospital, Goyang, ${ }^{5}$ Department of Statistics, Korea University, Seoul, ${ }^{6}$ Division of Endocrinology and Metabolism, Department of Internal Medicine, Yonsei University College of Medicine, Seoul, Korea

\section{Correspondence: Eun Jig Lee, MD, PhD}

Division of Endocrinology and Metabolism, Department of Internal Medicine,

Yonsei University College of Medicine,

50-1 Yonsei-ro, Seodaemun-gu, Seoul 03722, Korea

Tel: 82-2-2228-1983

Fax: 82-2-393-6884

E-mail: ejlee423@yuhs.ac

Received September 26, 2018

Accepted February 25, 2019

Published Online February 26, 2019

*Young Ki Lee and Kyeong Hye Park contributed equally to this work.

\begin{abstract}
Purpose
The volume of thyroid cancer screening and subsequent thyroid fine-needle aspiration (FNA) have rapidly increased in South Korea. We analyzed the thyroid cancer diagnoses/thyroid FNA ratio according to the annual number of FNA to evaluate changes in the diagnostic efficiency of FNA.
\end{abstract}

\section{Materials and Methods}

This was a nationwide population-based retrospective cohort study. The overall thyroid cancer diagnoses/thyroid FNA ratio and annual incremental thyroid cancer diagnoses/incremental thyroid FNA ratio were indirectly calculated using data obtained from the Korea Central Cancer Registry database and the Korean National Health Insurance Service claims database from 2004 to 2012. Pearson correlation analyses were performed to evaluate the strength of linear associations between variables.

\section{Results}

The number of thyroid FNA increased from 28,596 to 177,805 (6.2-fold increase) from 2004 to 2012 . The overall thyroid cancer diagnoses/thyroid FNA ratio decreased from $36.5 \%$ in 2004 to $25.1 \%$ in 2012 and was negatively correlated to the number of FNA $(R=-0.977, p<0.001)$. The annual incremental thyroid cancer diagnoses/incremental thyroid FNA ratios (range, $15.3 \%$ to $30.7 \%$ ) were always lower than the overall thyroid cancer diagnoses/thyroid FNA ratio in each year and also worsened according to the increase in the number of FNA $(R=-0.853, p=0.007)$.

\section{Conclusion}

The diagnostic performance of both overall and annual incremental thyroid FNA worsened, whereas the number of thyroid FNA procedures increased. More sophisticated indications for FNA are required to improve its diagnostic efficiency, considering the increased burden of screening-detected thyroid nodules.

\section{Introduction}

The incidence of thyroid cancer has significantly increased over the past two decades worldwide [1]. Several studies have shown that the increased implementation of various

\section{Key words}

Thyroid neoplasms, Fine-needle aspiration, Diagnostic efficiency, Screening imaging modalities has contributed to the increasing diagnoses of thyroid cancer [1-5]. This phenomenon is particularly remarkable in the Republic of Korea, where the incidence of thyroid cancer has increased nearly by 10 -fold from 1999 to 2013 [6].

Thyroid fine-needle aspiration (FNA) is the most impor- 
tant diagnostic test for detecting thyroid cancer [7]. Regarding the rapid increase in thyroid cancer, there is a growing concern that the number of FNA procedures has unnecessarily increased [8,9]. If FNA is performed unnecessarily in screening-detected thyroid nodules that have a low likelihood of cancer, the relative frequency of obtaining negative results could increase, and the diagnostic efficiency of FNA might decrease according to the increased volume of screening and subsequent FNA.

The diagnostic efficiency of a test may be assessed using the number of diagnoses of the index disease compared to the number of tests performed [10-14]. If the frequency of detecting a patient according to the test is reduced, the number of tests required to identify one patient would increase, resulting in an increase in the overall cost and potential adverse events [10-14]. Therefore, the diagnostic efficiency of FNA could be estimated using the ratio of thyroid cancer diagnoses and FNA procedures.

We assumed that the question of whether FNA for the diagnosis of thyroid cancer was performed efficiently could be answered by looking at recent changes in the ratio of thyroid cancer diagnoses and FNA procedures. In addition, we assumed that the ratio of incremental thyroid cancer diagnoses and incremental thyroid FNA biopsies would reflect the diagnostic efficiency of FNA for additionally detected thyroid nodules from the increased screening. Thus, to evaluate the change of diagnostic efficiency of FNA and the potential influence of the number of FNA biopsies to the changed efficacy, we analyzed the changes in overall and annual incremental thyroid cancer diagnoses/thyroid FNA ratio according to the annual number of FNA biopsies performed during the era of increased thyroid cancer screening in South Korea.

\section{Materials and Methods}

\section{Data sources}

This was a nationwide retrospective cohort study from 2004 to 2012 using two nationwide databases.

The number of thyroid FNA biopsies performed was obtained from the claims database of the National Health Insurance (NHI) system of the Republic of Korea [15]. The NHI system is a mandatory universal health insurance system that has been covering the entire population ( $>50$ million) in Korea since 1989. NHI covers all forms of health services, including admission, ambulatory care, and pharmaceutical services. The medical claims database stores data about the use of all healthcare services. Moreover, it contains extensive data, including demographic information such as age and sex, record of medical services such as diagnosis statements according to the International Classification of Diseases, 10th revision (ICD-10), detailed statements about prescriptions and surgical and other procedures such as thyroid FNA.

We used the Korea Central Cancer Registry (KCCR) database to obtain the data regarding thyroid cancer diagnoses [16]. The KCCR was initiated by the Ministry of Health and Welfare in Korea in 1980 and then expanded nationally to cover over $95 \%$ of all patients with newly diagnosed cancer in Korea during the study period.

\section{Case identification}

The information about the number of FNA biopsies was identified using the NHI claims code (C8591) in the NHI claims database. The number of thyroid FNA biopsies according to sex was obtained only from 2006 as claims data for thyroid FNA until 2005 were often missing sex information. The number of newly diagnosed patients with thyroid cancer was obtained from the KCCR database from 2004 to 2012.

\section{Overall thyroid cancer diagnoses/thyroid FNA ratio}

We considered that most thyroid FNA were performed to diagnose thyroid cancer, and most patients diagnosed with thyroid cancer underwent preoperative thyroid FNA. Since the NHI claims database can store all data on thyroid FNA performed in the Republic of Korea, the nationwide thyroid cancer diagnoses/thyroid FNA ratio could be indirectly estimated by dividing the number of incident cases of thyroid cancer in a year by the number of thyroid FNA in such year.

\section{Annual incremental thyroid cancer diagnoses/incremen- tal thyroid FNA ratio}

The annual increment of thyroid cancer diagnoses was calculated as the number of thyroid cancer diagnoses in each year minus the number of thyroid cancer diagnosis in the preceding year (e.g., annual increment of thyroid cancer diagnoses in $2010=$ the number of thyroid cancer diagnoses in 2010 - the number of thyroid cancer diagnoses in 2009). The annual increment of thyroid FNA biopsies for each year was calculated as the number of thyroid FNA biopsies in each year minus the number of thyroid FNA biopsies in the preceding year (e.g., annual increment of thyroid FNA biopsies in 2010=the number of thyroid FNA biopsies in 2010-the number of thyroid FNA biopsies in 2009). The annual incremental thyroid cancer diagnoses/incremental thyroid FNA 
Table 1. Number of thyroid FNA and thyroid cancer diagnoses, and estimated thyroid cancer diagnoses/thyroid FNA ratio

\begin{tabular}{|c|c|c|c|c|c|c|c|c|c|}
\hline \multirow[t]{2}{*}{ Year } & \multicolumn{3}{|c|}{ No. of FNA } & \multicolumn{3}{|c|}{$\begin{array}{l}\text { No. of thyroid } \\
\text { cancer diagnoses }\end{array}$} & \multicolumn{3}{|c|}{$\begin{array}{l}\text { Thyroid cancer diagnoses/thyroid } \\
\text { FNA ratio }(\%)^{\text {a) }}\end{array}$} \\
\hline & Total $^{\text {b) }}$ & Male & Female & Total & Male & Female & Total $^{\text {b) }}$ & Male & Female \\
\hline 2004 & 28,596 & $\mathrm{~N} / \mathrm{A}$ & $\mathrm{N} / \mathrm{A}$ & 10,424 & 1,417 & 9,007 & 36.5 & $\mathrm{~N} / \mathrm{A}$ & $\mathrm{N} / \mathrm{A}$ \\
\hline 2005 & 37,781 & $\mathrm{~N} / \mathrm{A}$ & $\mathrm{N} / \mathrm{A}$ & 12,793 & 1,781 & 11,012 & 33.9 & $\mathrm{~N} / \mathrm{A}$ & $\mathrm{N} / \mathrm{A}$ \\
\hline 2006 & 48,720 & 7,018 & 41,677 & 16,152 & 2,332 & 13,820 & 33.2 & 33.2 & 33.2 \\
\hline 2007 & 69,110 & 10,167 & 58,922 & 21,283 & 3,187 & 18,096 & 30.8 & 31.3 & 30.7 \\
\hline 2008 & 89,821 & 13,865 & 75,939 & 27,324 & 4,337 & 22,987 & 30.4 & 31.3 & 30.3 \\
\hline 2009 & 110,393 & 17,844 & 92,522 & 32,507 & 5,239 & 27,268 & 29.4 & 29.4 & 29.5 \\
\hline 2010 & 133,368 & 22,155 & 111,190 & 36,767 & 6,351 & 30,416 & 27.6 & 28.7 & 27.4 \\
\hline 2011 & 155,987 & 25,428 & 130,535 & 41,289 & 7,110 & 34,179 & 26.5 & 28.0 & 26.2 \\
\hline 2012 & 177,805 & 30,002 & 147,774 & 44,621 & 8,154 & 36,467 & 25.1 & 27.2 & 24.7 \\
\hline
\end{tabular}

FNA, fine-needle aspiration; N/A, not assessed. a)Thyroid cancer diagnoses/thyroid FNA ratio was estimated by dividing the number of newly diagnosed patients with thyroid cancer in a year by the number of thyroid FNA in such year, ${ }^{\text {b) The }}$ total number included cases in which the sex of a participant was not identified.

ratio was defined as the annual increment of thyroid cancer diagnoses divided by the annual increment of thyroid FNA biopsies.

\section{Statistical analysis}

Pearson correlation coefficients were calculated to evaluate whether the number of thyroid cancer diagnoses and the thyroid cancer diagnoses/thyroid FNA ratio were linearly associated with the annual number of FNA. All statistical analyses were performed using the Statistical Package for the Social Sciences software ver. 23.0 for Windows (IBM Corp., Armonk, NY). All tests were two-sided, and a p-value of $<0.05$ was considered statistically significant.

\section{Ethical statement}

Personal identifiable information in the medical records was de-identified to comply with the Health Insurance Portability and Accountability Act privacy rule. In addition, since the information in the NHI database is encrypted, the database does not contain personal identifiers. The study was approved by the Institutional Review Board of Ilsan Hospital (IRB No. NHIMC 2016-02-007) and performed in accordance with the principles of the Declaration of Helsinki. The informed consent was waived.

\section{Results}

\section{Number of annual thyroid FNA and thyroid cancer diag- noses}

Table 1 shows the number of thyroid FNA and thyroid cancer diagnoses from 2004 to 2012. The number of thyroid FNA increased from 28,596 to 177,805 (6.2-fold increase) from 2004 to 2012. During the same period, the number of newly diagnosed patients with thyroid cancer increased from 10,424 to 44,621 (4.3-fold increase). A strong positive linear correlation was observed between the number of thyroid FNA and the number of thyroid cancer diagnoses $(R=0.995$, $\mathrm{p}<0.001$ ) (Fig. 1A).

\section{Overall thyroid cancer diagnoses/thyroid FNA ratio according to the passage of time and number of FNA}

The overall thyroid cancer diagnoses/thyroid FNA ratio gradually decreased for 8 years from $36.5 \%$ in 2004 to $25.1 \%$ in 2012 (Table 1). A strong negative correlation was observed between the number of thyroid FNA and the overall thyroid cancer diagnoses / thyroid FNA ratio $(\mathrm{R}=-0.977, \mathrm{p}<0.001)$ (Fig. 1B).

\section{Annual incremental thyroid cancer diagnoses/incremen- tal thyroid FNA ratio according to the passage of time and number of FNA}

Table 2 shows the incremental number of FNA, increment in thyroid cancer diagnoses, and annual incremental thyroid 

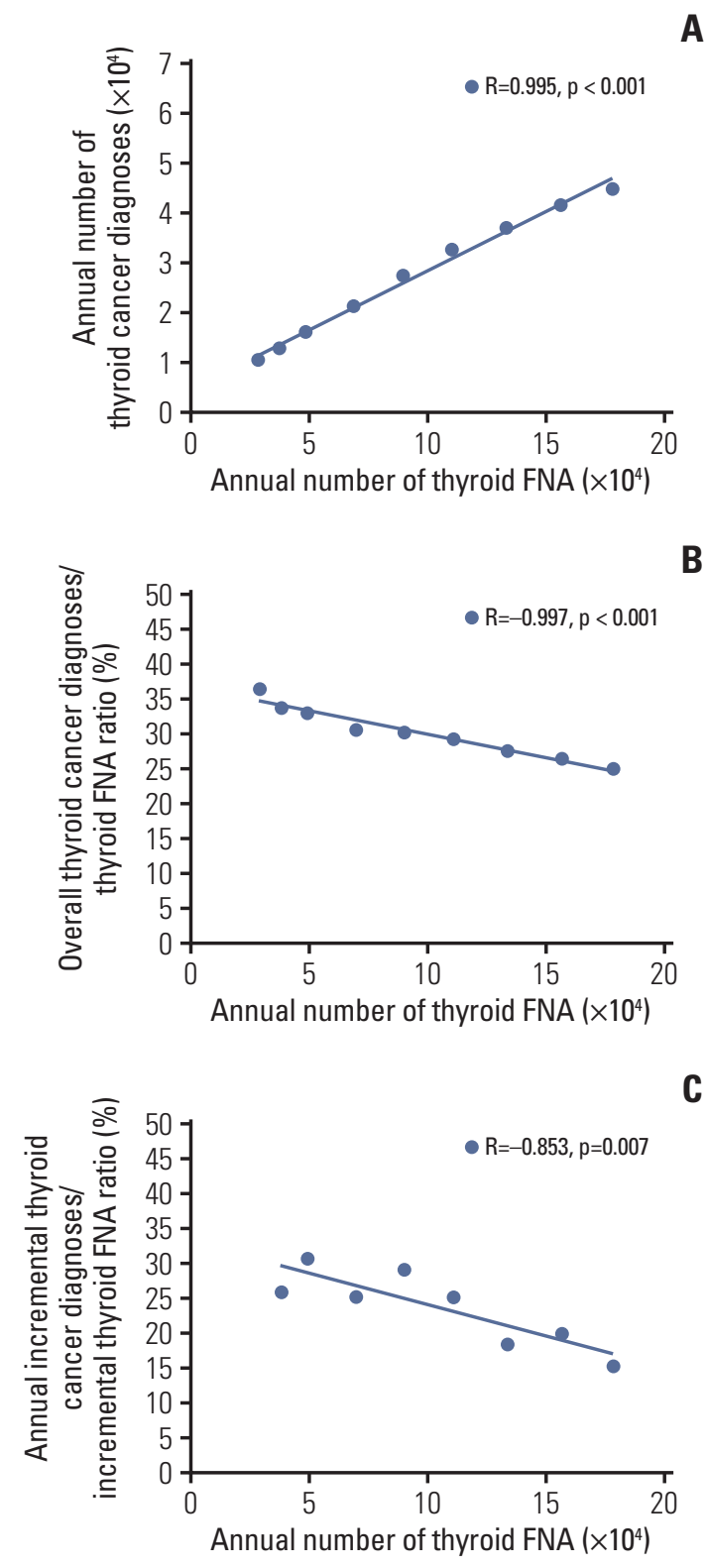

Fig. 1. Pearson correlation coefficients were calculated to evaluate the association between the annual number of thyroid fine-needle aspiration (FNA) and the annual number of thyroid cancer diagnoses $(\mathrm{A})$, overall thyroid cancer diagnoses / thyroid FNA ratio (B), and annual incremental thyroid cancer diagnoses / incremental thyroid FNA ratio (C). The lines indicate significant correlations $(\mathrm{p}<0.05)$.

cancer diagnoses/incremental thyroid FNA ratio (which were assumed to reflect FNA for screening-detected thyroid nodule) from 2005 to 2012. A negative correlation was observed between the number of thyroid FNA biopsies and the annual incremental thyroid cancer diagnoses / incremen- tal thyroid FNA ratio ( $\mathrm{R}=-0.853, \mathrm{p}=0.007)$ (Fig. 1C). During the entire research period, each annual incremental thyroid cancer diagnoses / incremental thyroid FNA ratio was lower than the overall thyroid cancer diagnoses/thyroid FNA ratio in the same year.

\section{Discussion}

In this study, we showed that the diagnostic efficiency of the overall and annual incremental thyroid FNA estimated by the thyroid cancer diagnoses/thyroid FNA ratio decreased as the annual number of thyroid FNA increased from 2004 to 2012.

The rapid increase in the incidence of thyroid cancer is largely attributed to the increase in thyroid cancer screening [1-5]. Udelsman and Zhang [2] showed a linear correlation between the frequency of neck ultrasonography and the incidence of thyroid cancer in the United States. Similar linear correlations were observed between the annual number of computed tomography imaging and incidence of thyroid cancer [3]. In addition, imaging procedures, such as Doppler examinations of the neck vessels and positron emission tomography scans, have increased the detection of incidental thyroid nodules $[17,18]$. As most of the nodules found in screening are confirmed as benign [19], many experts are concerned that increased thyroid cancer screening may lead to an increase in unnecessary FNA biopsies and a decrease in the diagnostic efficiency of FNA [20].

In this study, the number of thyroid FNA biopsies was positively correlated with the number of thyroid cancer diagnoses. However, the overall thyroid cancer diagnoses / thyroid FNA ratio decreased as the annual number of FNA increased. Our findings are in line with previous studies. In a study using U.S. Veterans Affairs administrative data from 2000 to 2012, the authors suggested that the increased thyroid cancer incidence may be related to increased use of thyroid ultrasound and FNA, based on their simultaneous increase [9]. Notably, the use of FNA increased much more rapidly than the incidence of thyroid cancer in the study, and the incidence increased only 2-fold while the use of FNA increased 7-fold [9]. In another study using an NHI sample cohort that includes approximately $2.2 \%$ of the entire Korean population, a higher frequency of thyroid FNA in a region was associated with a lower possibility of thyroid cancer diagnosis in patients who underwent thyroid FNA in the region [20]. Altogether, the diagnostic efficiency of FNA seems to be decreased when the frequency of thyroid FNA biopsies increases.

To further explore the cause of the decline in the diagnostic 
Table 2. Estimated incremental thyroid cancer diagnoses/incremental thyroid FNA ratio

\begin{tabular}{|c|c|c|c|c|c|c|c|c|c|}
\hline \multirow[t]{2}{*}{ Year } & \multicolumn{3}{|c|}{ Increment of thyroid FNA } & \multicolumn{3}{|c|}{$\begin{array}{l}\text { Increment of thyroid } \\
\left.\text { cancer diagnoses }{ }^{\mathrm{b}}\right)\end{array}$} & \multicolumn{3}{|c|}{$\begin{array}{l}\text { Incremental thyroid cancer diagnoses/ } \\
\text { incremental thyroid FNA ratio }(\%)^{c}\end{array}$} \\
\hline & Total $^{\mathrm{d})}$ & Male & Female & Total $^{(\mathrm{d})}$ & Male & Female & Total $^{\mathrm{d})}$ & Male & Female \\
\hline 2005 & 9,185 & $\mathrm{~N} / \mathrm{A}$ & $\mathrm{N} / \mathrm{A}$ & 2,369 & 364 & 2,005 & 25.8 & $\mathrm{~N} / \mathrm{A}$ & $\mathrm{N} / \mathrm{A}$ \\
\hline 2006 & 10,939 & $\mathrm{~N} / \mathrm{A}$ & $\mathrm{N} / \mathrm{A}$ & 3,359 & 551 & 2,808 & 30.7 & $\mathrm{~N} / \mathrm{A}$ & $\mathrm{N} / \mathrm{A}$ \\
\hline 2007 & 20,390 & 3,149 & 17,245 & 5,131 & 855 & 4,276 & 25.2 & 27.2 & 24.8 \\
\hline 2008 & 20,711 & 3,698 & 17,017 & 6,041 & 1,150 & 4,891 & 29.2 & 31.1 & 28.7 \\
\hline 2009 & 20,572 & 3,979 & 16,583 & 5,183 & 902 & 4,281 & 25.2 & 22.7 & 25.8 \\
\hline 2010 & 22,975 & 4,311 & 18,668 & 4,260 & 1,112 & 3,148 & 18.5 & 25.8 & 16.9 \\
\hline 2011 & 22,619 & 3,273 & 19,345 & 4,522 & 759 & 3,763 & 20.0 & 23.2 & 19.5 \\
\hline 2012 & 21,818 & 4,574 & 17,239 & 3,332 & 1,044 & 2,288 & 15.3 & 22.8 & 13.3 \\
\hline
\end{tabular}

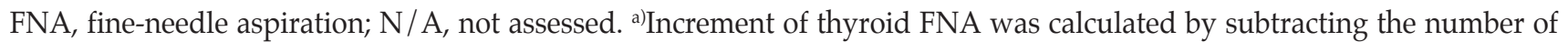
FNA in the previous year from that in the index year, ${ }^{\mathrm{b}}$ Increment of thyroid cancer diagnoses was calculated by subtracting the number of thyroid cancer diagnoses in the last year from that in the index year, ${ }^{\mathrm{c}}$ Incremental thyroid cancer diagnoses/ incremental thyroid FNA ratio was calculated by dividing the increment of thyroid cancer diagnoses by the increment of thyroid FNA, diThe total number included cases in which the sex of a participant was not identified.

efficiency of FNA, we assumed that the incremental thyroid cancer diagnoses/incremental thyroid FNA ratio would indirectly reflect the diagnostic efficiency of FNA for additionally detected thyroid nodules from the increased screening. If the increase in the number of FNA biopsies and thyroid cancer diagnoses is largely attributed to the increase in the frequency of screening, the increment of thyroid FNA would reflect the number of FNA biopsies performed for additionally detected nodules from the increase in the frequency of screening (i.e., additional screening-detected thyroid nodules). In addition, the increment of thyroid cancer diagnoses would reflect the number of cancer diagnoses from the additional screening-detected thyroid nodules. We found that the annual incremental thyroid cancer diagnoses/incremental thyroid FNA ratio was lower than the overall thyroid cancer diagnoses/thyroid FNA ratio in each year. Moreover, the annual incremental thyroid cancer diagnoses/incremental thyroid FNA ratio decreased as the annual number of FNA biopsies increased. Therefore, the efficiency of the overall thyroid FNA appears to have been compromised during the period of increasing thyroid cancer screening as the diagnostic efficiency of FNA for screeningdetected nodules was low and had decreased gradually. Our estimates of the annual incremental thyroid cancer diagnoses/incremental thyroid FNA ratio (range, 15.3\% to 30.7\%) are in accordance with the thyroid cancer diagnoses/thyroid FNA ratio in screening-detected nodules obtained by another study in Korea. In the study from a single institution, 111 cases of ultrasound-guided FNA were performed in the process of identifying 29 thyroid cancers from screeningdetected thyroid nodules (thyroid cancer diagnoses / thyroid
FNA ratio, 26.1\%) [19].

Although thyroid FNA is relatively safe and inexpensive (\$250 in the Republic of Korea and \$500 in the United States $[21,22])$, the survival benefit of thyroid cancer screening program was low or even unclear $[23,24]$. A low diagnostic efficiency increases the number of FNA performed in the course of diagnosing a patient; thereby, it could increase the risk compared to the benefit of the test. Thus, the higher the number of screening-detected thyroid nodules, the higher the need for more sophisticated indications for FNA to improve its diagnostic efficiency and the balance between the risks and benefits.

This study has several strengths. First, this is the largest study to date of the relationship between FNA frequency and the number of thyroid cancer diagnoses using the NHI database including the entire Korean population. Second, to the best of our knowledge, this is the first study that suggests the worsened performance of FNA in screening-detected nodule caused by the increase in the frequency of FNA biopsies. Third, we overcame the limitations of the NHI database on the accuracy of diagnosis by using the KCCR database to assess the number of patients with thyroid cancer. Diagnostic accuracy is a common limitation in using health administrative data, although the reliability of the information in the NHI database has been previously validated in several studies [25-29].

This study also has several limitations. First, the number of patients with newly diagnosed thyroid cancer in this study may be slightly underestimated. The KCCR data are usually based on postoperative pathology results. Therefore, some patients with thyroid cancer who were followed up without 
surgery after FNA (active surveillance or inappropriate condition for operation) may be missing in this study. Second, there may be cases in which thyroid FNA was used for purposes other than the diagnosis of thyroid cancer and thyroid cancer was diagnosed without thyroid FNA. However, the diagnosis of thyroid cancer without performing FNA is rare. Third, several technological advances related to thyroid FNA, as well as training level and case volume of physicians, may influence the diagnostic efficiency of thyroid FNA [30-32]. However, the training level and case volume of physicians are likely to be enhanced according to the increased nationwide frequency of thyroid FNA, which would potentially improve diagnostic accuracy, just as the technological advances would. Our results suggest that the diagnostic efficiency of FNA was compromised despite the potential enhancement of its accuracy. Fourth, the exact volume of thyroid cancer screening could not be assessed since NHI did not cover thyroid ultrasonography during the study period. Thyroid cancer screening during the study period was often performed via a health screening program that was not covered by NHI. Thus, the diagnostic efficiency of FNA for screening-detected nodules was indirectly estimated based on several assumptions, even though the estimate was similar to the results obtained in the other study.
Fifth, we could not determine the characteristics of the nodules targeted by FNA because of the absence of information about sonographic findings in the NHI database.

In conclusion, the overall diagnostic efficiency of FNA worsened according to the increase in the number of FNA, with a low and decreased diagnostic efficiency of incremental FNA. Our findings suggest that more sophisticated indications for FNA are required to improve its diagnostic efficiency, considering the burden of screening-detected thyroid nodules.

\section{Conflicts of Interest}

Conflict of interest relevant to this article was not reported.

\section{Acknowledgments}

The authors express their gratitude to Hyunsun Lim (Research Institute, National Health Insurance Service Ilsan Hospital) for providing statistical advice.

This study used the health administration data of the policy report published by National Health Insurance Service Ilsan Hospital (NHIS-2014-1-022).

\section{References}

1. Global Burden of Disease Cancer Collaboration, Fitzmaurice C, Dicker D, Pain A, Hamavid H, Moradi-Lakeh M, et al. The Global Burden of Cancer 2013. JAMA Oncol. 2015;1:505-27.

2. Udelsman R, Zhang Y. The epidemic of thyroid cancer in the United States: the role of endocrinologists and ultrasounds. Thyroid. 2014;24:472-9.

3. Hoang JK, Choudhury KR, Eastwood JD, Esclamado RM, Lyman GH, Shattuck TM, et al. An exponential growth in incidence of thyroid cancer: trends and impact of CT imaging. AJNR Am J Neuroradiol. 2014;35:778-83.

4. Vaccarella S, Dal Maso L, Laversanne M, Bray F, Plummer M, Franceschi S. The impact of diagnostic changes on the rise in thyroid cancer incidence: a population-based study in selected high-resource countries. Thyroid. 2015;25:1127-36.

5. Oh CM, Jung KW, Won YJ, Shin A, Kong HJ, Lee JS. Ageperiod-cohort analysis of thyroid cancer incidence in Korea. Cancer Res Treat. 2015;47:362-9.

6. Park S, Oh CM, Cho H, Lee JY, Jung KW, Jun JK, et al. Association between screening and the thyroid cancer "epidemic" in South Korea: evidence from a nationwide study. BMJ. 2016; 355:i5745.

7. Haugen BR, Alexander EK, Bible KC, Doherty GM, Mandel SJ, Nikiforov YE, et al. 2015 American Thyroid Association man- agement guidelines for adult patients with thyroid nodules and differentiated thyroid cancer: the American Thyroid Association Guidelines Task Force on Thyroid Nodules and Differentiated Thyroid Cancer. Thyroid. 2016;26:1-133.

8. Ahn HS, Kim HJ, Welch HG. Korea's thyroid-cancer "epidemic": screening and overdiagnosis. N Engl J Med. 2014;371:1 765-7.

9. Zevallos JP, Hartman CM, Kramer JR, Sturgis EM, Chiao EY. Increased thyroid cancer incidence corresponds to increased use of thyroid ultrasound and fine-needle aspiration: a study of the Veterans Affairs health care system. Cancer. 2015;121: 741-6.

10. Zimetbaum PJ, Kim KY, Josephson ME, Goldberger AL, Cohen DJ. Diagnostic yield and optimal duration of continuous-loop event monitoring for the diagnosis of palpitations: a cost-effectiveness analysis. Ann Intern Med. 1998;128:890-5.

11. Steinberg LA, Knilans TK. Syncope in children: diagnostic tests have a high cost and low yield. J Pediatr. 2005;146:355-8.

12. Valencia CA, Husami A, Holle J, Johnson JA, Qian Y, Mathur A, et al. Clinical impact and cost-effectiveness of whole exome sequencing as a diagnostic tool: a pediatric center's experience. Front Pediatr. 2015;3:67.

13. Bini EJ, Cohen J. Diagnostic yield and cost-effectiveness of 
endoscopy in chronic human immunodeficiency virus-related diarrhea. Gastrointest Endosc. 1998;48:354-61.

14. Larson EB, Omenn GS, Lewis H. Diagnostic evaluation of headache: impact of computerized tomography and costeffectiveness. JAMA. 1980;243:359-62.

15. Song SO, Jung CH, Song YD, Park CY, Kwon HS, Cha BS, et al. Background and data configuration process of a nationwide population-based study using the Korean national health insurance system. Diabetes Metab J. 2014;38:395-403.

16. Shin HR, Won YJ, Jung KW, Kong HJ, Yim SH, Lee JK, et al. Nationwide cancer incidence in Korea, 1999 2001: first result using the national cancer incidence database. Cancer Res Treat. 2005;37:325-31.

17. Nilsson IL, Arnberg F, Zedenius J, Sundin A. Thyroid incidentaloma detected by fluorodeoxyglucose positron emission tomography / computed tomography: practical management algorithm. World J Surg. 2011;35:2691-7.

18. Steele SR, Martin MJ, Mullenix PS, Azarow KS, Andersen CA. The significance of incidental thyroid abnormalities identified during carotid duplex ultrasonography. Arch Surg. 2005;140: 981-5.

19. Yoo J, Ahn HS, Kim SJ, Park SH, Seo M, Chong S. Evaluation of diagnostic performance of screening thyroid ultrasonography and imaging findings of screening-detected thyroid cancer. Cancer Res Treat. 2018;50:11-8.

20. Cho YJ, Kim DY, Park EC, Han KT. Thyroid fine-needle aspiration biopsy positively correlates with increased diagnosis of thyroid cancer in South Korean patients. BMC Cancer. 2017; $17: 114$.

21. Heller M, Zanocco K, Zydowicz S, Elaraj D, Nayar R, Sturgeon C. Cost-effectiveness analysis of repeat fine-needle aspiration for thyroid biopsies read as atypia of undetermined significance. Surgery. 2012;152:423-30.

22. Yip L, Farris C, Kabaker AS, Hodak SP, Nikiforova MN, McCoy KL, et al. Cost impact of molecular testing for indeterminate thyroid nodule fine-needle aspiration biopsies. J Clin Endocrinol Metab. 2012;97:1905-12.

23. Lin JS, Bowles EJ, Williams SB, Morrison CC. Screening for thyroid cancer: updated evidence report and systematic review for the US Preventive Services Task Force. JAMA. 2017;317:1888-903.

24. Choi YM, Kim WG, Kwon H, Jeon MJ, Han M, Kim TY, et al. Changes in standardized mortality rates from thyroid cancer in Korea between 1985 and 2015: Analysis of Korean national data. Cancer. 2017;123:4808-14.

25. Park SK, Cheong HK, Ki M, Son YM, Kim H. Assessment of the availability of health insurance data for epidemiologic study of childhood aseptic meningitis. Korean J Prev Med. 2003;36:349-58.

26. Cheong HK, Park SK, Ki M, Lee K. Six year national trend of childhood aseptic meningitis incidence in Korea, 1996-2001. Korean J Epidemiol. 2008;30:252-62.

27. Park BJ, Sung JH, Park KD, Seo SW, Kim SW. Report of the evaluation for validity of discharged diagnoses in Korean Health Insurance database. Seoul: Seoul National University; 2003. p. 19-52.

28. Lee SY, Chung SE, Kim DW, Eun SH, Kang HC, Cho YW, et al. Estimating the prevalence of treated epilepsy using administrative health data and its validity: ESSENCE Study. J Clin Neurol. 2016;12:434-40.

29. Kimm H, Yun JE, Lee SH, Jang Y, Jee SH. Validity of the diagnosis of acute myocardial infarction in Korean national medical health insurance claims data: the Korean heart study (1). Korean Circ J. 2012;42:10-5.

30. Houlton JJ, Sun GH, Fernandez N, Zhai QJ, Lucas F, Steward DL. Thyroid fine-needle aspiration: does case volume affect diagnostic yield and interpretation? Arch Otolaryngol Head Neck Surg. 2011;137:1136-9.

31. Beland MD, Anderson TJ, Atalay MK, Grand DJ, Cronan JJ. Resident experience increases diagnostic rate of thyroid fineneedle aspiration biopsies. Acad Radiol. 2014;21:1490-4.

32. Lee YH, Baek JH, Jung SL, Kwak JY, Kim JH, Shin JH, et al. Ultrasound-guided fine needle aspiration of thyroid nodules: a consensus statement by the korean society of thyroid radiology. Korean J Radiol. 2015;16:391-401. 